\title{
Checking, processing and verification of nuclear data covariances
}

\author{
Oscar Cabellos ${ }^{*}$, James Dyrda, and Nicolas Soppera \\ OECD NEA, Boulogne-Billancourt, France \\ Received: 30 October 2017 / Received in final form: 17 January 2018 / Accepted: 14 May 2018
}

\begin{abstract}
The aim of this paper is to present the activities carried out by NEA Data Bank on checking, processing and verification of JEFF-3.3T4 covariances. A picture of the completeness and status of the JEFF$3.3 \mathrm{~T} 4$ covariances is addressed. The verification of JEFF-3.3T4 covariances is performed with nuclear data sensitivity tool providing the $k_{\text {eff }}$ uncertainty as a function of the contributing nuclide-reaction pairings including cross-reaction covariances. A total number of 4501 ICSBEP benchmarks is used in this analysis. This exercise is also extended to covariance libraries such as JENDL-4.0 updated files, ENDF/B-VII.1, SCALE-6.2rev8 and ENDF/B-VIII.0 $\beta 5$, allowing comparison of these results with both the experimental criticality benchmark and different methodologies of evaluation.
\end{abstract}

\section{Introduction}

JEFF-3.3T4 [1] is a fully consistent and complete nuclear data library with all data needed and associated covariance information (full covariance information for the main actinides), which can be reliably used for a large spectrum of applications, and which has shown a better performance than JEFF-3.2 library [2]. Previous work carried out by NEA Data Bank on checking, processing and verification of earlier JEFF-3.3 beta releases has been presented in past JEFF meetings [3,4]. In this paper, the efforts of NEA Data Bank focused on the latest beta JEFF-3.3T4 release are presented. A review of NEA tools and databases is described in Section 2.

In Section 3, we have performed a comparison of relative standard deviation and correlation matrix with major covariance nuclear data libraries such as ENDF/BVII.1 [5], JENDL-4.0u1 [6], SCALE6.2 [7] and the recent one CIELO1(ENDF/B-VIII.0. $\beta 5$ ) [8]. This information gives a good indication of the current status of JEFF-3.3T4 covariances. A thorough review of the current covariance data files associated with the latest versions of the JENDL4.0 updated files (JENDL-4.0u) and ENDF/B-VII.1 evaluated data files can be seen in reference [9].

Checking and processing issues are remarked in Section 4. After some checking tests of ENDF6 format (e.g. consistency between MF2 and MF32, energy ranges, etc.) and mathematical verification (e.g. positive definite matrix, abnormal values, etc.) few problems in raw covariances were noted. The complete set of JEFF-3.3T4

\footnotetext{
* e-mail: oscar.cabellos@upm.es
}

covariances (MF31, MF32/MF33, MF34 and MF35) are processed using the code NJOY2012.99 [10]; formatting and processing issues will be discussed in the paper.

The performance of JEFF-3.3T4 covariance library in criticality and safety analysis is outlined in Section 5 . Nuclear data sensitivity tool (NDaST) [11] is able to propagate the covariance of nuclear data in 4501 ICSBEP benchmarks allowing to address this question in different fissile materials and neutron spectrum. This work is extended to the whole nuclear data library with especial emphasis on the four major actinides, ${ }^{233} \mathrm{U},{ }^{235} \mathrm{U},{ }^{238} \mathrm{U}$, and ${ }^{239} \mathrm{Pu}$. Finally, a summary of the criticality uncertainty results is given in Tables $1-4$, showing the impact and differences of current covariance nuclear data evaluations.

\section{NEA tools and databases}

The processing and verification of nuclear data covariances have been performed with the NEA tools and databases. These tools and databases are extensively used by the nuclear data community being an essential part of the Nuclear Data Services delivered by the NEA. Hereafter, a brief summary of these tools is presented emphasising the main features on nuclear data covariances:

- The java-based nuclear information software (JANIS) [12] software developed by the NEA Data Bank is used to facilitate the visualisation and manipulation of nuclear data, giving access to evaluated nuclear data libraries, such as ENDF, JEFF, JENDL, TENDL, etc. JANIS is able to read different covariance formats: ENDF, COVERX, ERRORR and BOXER. 
Table 1. Impact of different ${ }^{235} \mathrm{U}$ nuclear data covariances in ICSBEP suite averaged for fissile material and spectrum.

\begin{tabular}{|c|c|c|c|c|c|c|c|c|c|c|c|c|}
\hline & \multirow{2}{*}{$\begin{array}{l}\text { Fiss. mat. } \\
\text { Spect. } \\
\text { Benchm.\# } \\
\text { Exp. unc. }\end{array}$} & \multicolumn{4}{|c|}{$\mathrm{HEU}$} & \multicolumn{4}{|c|}{ IEU } & \multicolumn{3}{|c|}{ LEU } \\
\hline & & $\begin{array}{l}\text { Fast } \\
463 \\
210\end{array}$ & $\begin{array}{l}\text { Inter. } \\
21 \\
343\end{array}$ & $\begin{array}{l}\text { Mix. } \\
78 \\
419\end{array}$ & $\begin{array}{l}\text { Therm. } \\
802 \\
468\end{array}$ & $\begin{array}{l}\text { Fast } \\
57 \\
177\end{array}$ & $\begin{array}{l}\text { Inter. } \\
8 \\
203\end{array}$ & $\begin{array}{l}\text { Mix. } \\
8 \\
311\end{array}$ & $\begin{array}{l}\text { Therm. } \\
142 \\
511\end{array}$ & $\begin{array}{l}\text { Fast } \\
1 \\
270\end{array}$ & $\begin{array}{l}\text { Mix. } \\
5 \\
348\end{array}$ & $\begin{array}{l}\text { Therm } \\
1512 \\
259\end{array}$ \\
\hline \multirow[t]{4}{*}{ E-VIII.0b5 } & Summed & 1012 & 1440 & 1100 & 756 & 934 & 1277 & 1168 & 753 & 1001 & 952 & 633 \\
\hline & $\mathrm{XSs}$ & 778 & 601 & 504 & 285 & 724 & 574 & 357 & 311 & 724 & 572 & 290 \\
\hline & Nubar & 631 & 1288 & 959 & 651 & 570 & 1124 & 1096 & 618 & 689 & 756 & 550 \\
\hline & PFNS & 73 & 151 & 163 & 219 & 130 & 111 & 165 & 285 & 59 & 51 & 102 \\
\hline \multirow[t]{4}{*}{$\mathrm{J}-3.3 \mathrm{~T} 4$} & Summed & 1190 & 1257 & 946 & 1043 & 1407 & 1313 & 883 & 1188 & 1084 & 916 & 720 \\
\hline & $\mathrm{XSs}$ & 1036 & 1053 & 676 & 330 & 1158 & 1099 & 539 & 339 & 903 & 708 & 328 \\
\hline & Nubar & 514 & 544 & 538 & 560 & 444 & 496 & 529 & 557 & 505 & 501 & 535 \\
\hline & PFNS & 236 & 347 & 347 & 755 & 654 & 435 & 433 & 987 & 323 & 290 & 313 \\
\hline \multirow[t]{4}{*}{ E-VII.1 } & Summed & 1345 & 2032 & 1277 & 979 & 1498 & 1786 & 917 & 1086 & 1261 & 1025 & 752 \\
\hline & $\mathrm{XSs}$ & 1200 & 1877 & 1077 & 232 & 1325 & 1606 & 594 & 256 & 1116 & 861 & 237 \\
\hline & Nubar & 543 & 598 & 548 & 660 & 480 & 548 & 552 & 663 & 532 & 509 & 647 \\
\hline & PFNS & 217 & 342 & 356 & 624 & 490 & 351 & 398 & 813 & 248 & 216 & 273 \\
\hline \multirow[t]{4}{*}{$\mathrm{J}-4.0 \mathrm{u} 1$} & Summed & 679 & 680 & 587 & 667 & 602 & 584 & 498 & 782 & 523 & 427 & 444 \\
\hline & $\mathrm{XSs}$ & 614 & 576 & 459 & 219 & 403 & 456 & 294 & 240 & 444 & 357 & 232 \\
\hline & Nubar & 243 & 182 & 177 & 289 & 204 & 174 & 197 & 290 & 222 & 183 & 283 \\
\hline & PFNS & 134 & 291 & 303 & 523 & 381 & 284 & 336 & 682 & 164 & 146 & 228 \\
\hline \multirow[t]{4}{*}{ S-6.2rev8 } & Summed & 1189 & 1917 & 1145 & 786 & 1408 & 1683 & 765 & 918 & 1136 & 888 & 519 \\
\hline & $\mathrm{XSs}$ & 1167 & 1859 & 1063 & 230 & 1323 & 1593 & 586 & 255 & 1112 & 856 & 236 \\
\hline & Nubar & 89 & 146 & 164 & 367 & 77 & 143 & 250 & 366 & 80 & 121 & 357 \\
\hline & PFNS & 192 & 337 & 352 & 611 & 463 & 337 & 392 & 797 & 215 & 198 & 267 \\
\hline
\end{tabular}

- The nuclear data evaluation cycle (NDEC) [13] is used to process the JEFF-3.3T4 and ENDF/B-VIII.0 $\beta 5$ files. At the end of the processing NDEC produces two files, one HENDF and one BOXER. These files are then uploaded into JANIS database using the "Import Wizard" tool to create a new database. JANIS can also import directly covariance evaluations, such as SCALE-6.2 in COVERX format. For a comparison with other evaluations (e.g. ENDF/B-VII.1 and JENDL-4.0u) the NEA database is used. This NEA database provides covariances in ENDF and BOXER format for many libraries, although some important covariances are missed, such as neutron multiplicity data (nubar) and prompt fission neutron spectrum (PFNS or Chi). In this work, we have processed nubar and PFNS for ENDF/B-VII.1 and JENDL-4.0u adding the processed covariances to NEA database.

- The 2016 database for the international criticality safety benchmark evaluation project (DICE) contains 567 evaluations representing 4874 critical, near-critical, or subcritical configurations into a standardised format that allows criticality safety analysis. This database is easily used to validate calculation tools and perform benchmarking to assess the performance of evaluated nuclear data libraries. DICE provides access to sensitivity coefficients (percent changes of $k$-effective due to elementary change of basic nuclear data) for the major nuclides and nuclear processes in a 30-group and 238group energy structure for 4501 experimental configurations.

- The NDaST is a Java based software, designed to perform calculations on nuclear data sensitivity files for benchmark cases. Here, NDaST is used for the calculation of the $k_{\text {eff }}$ uncertainty due to evaluated nuclear covariance data. This allows simple and fast analysis for nuclear data evaluators to test the impact of nuclear data covariances across the 4501 ICSBEP benchmarks with sensitivities in DICE. This tool is able to predict the impact of different evaluated covariances of individual nuclides and cross-sections (e.g., elastic, inelastic, fission, capture, their cross-correlations, etc.), nubar and PFNS.

In order to more easily facilitate the input of covariances to NDaST, an automated link has been introduced to the JANIS nuclear data viewing software. From a search dialogue within the "Covariances" panel of NDaST, the user may search their public/private JANIS covariance databases for a given nuclide and reaction combination. Selection of covariance format BOXER, ENDF or COVERX returned from this search allows users to quickly calculate the propagated nuclear data uncertainty. 
Table 2. Impact of different ${ }^{238} \mathrm{U}$ nuclear data covariances in ICSBEP suite averaged for fissile material and spectrum.

\begin{tabular}{|c|c|c|c|c|c|c|c|c|c|c|c|c|}
\hline & \multirow{2}{*}{$\begin{array}{l}\text { Fiss. mat. } \\
\text { Spect. } \\
\text { Benchm.\# } \\
\text { Exp. unc. }\end{array}$} & \multicolumn{4}{|c|}{$\mathrm{HEU}$} & \multicolumn{4}{|c|}{ IEU } & \multicolumn{3}{|c|}{ LEU } \\
\hline & & $\begin{array}{l}\text { Fast } \\
463 \\
210\end{array}$ & $\begin{array}{l}\text { Inter. } \\
21 \\
343\end{array}$ & $\begin{array}{l}\text { Mix. } \\
78 \\
419\end{array}$ & $\begin{array}{l}\text { Therm. } \\
802 \\
468\end{array}$ & $\begin{array}{l}\text { Fast } \\
57 \\
177\end{array}$ & $\begin{array}{l}\text { Inter. } \\
8 \\
203\end{array}$ & $\begin{array}{l}\text { Mix. } \\
8 \\
311\end{array}$ & $\begin{array}{l}\text { Therm. } \\
142 \\
511\end{array}$ & $\begin{array}{l}\text { Fast } \\
1 \\
270\end{array}$ & $\begin{array}{l}\text { Mix. } \\
5 \\
348\end{array}$ & $\begin{array}{l}\text { Therm } \\
1512 \\
259\end{array}$ \\
\hline \multirow[t]{4}{*}{ E-VIII.0b5 } & Summed & 49 & 38 & 12 & 13 & 428 & 369 & 154 & 92 & 124 & 227 & 291 \\
\hline & $\mathrm{XSs}$ & 45 & 37 & 10 & 13 & 366 & 342 & 143 & 91 & 116 & 215 & 285 \\
\hline & Nubar & 17 & 7 & 6 & 0 & 194 & 126 & 55 & 9 & 42 & 73 & 57 \\
\hline & PFNS & 6 & 2 & 1 & 0 & 101 & 38 & 15 & 5 & 8 & 11 & 9 \\
\hline \multirow[t]{4}{*}{$\mathrm{J}-3.3 \mathrm{~T} 4$} & Summed & 108 & 77 & 30 & 6 & 697 & 444 & 270 & 73 & 235 & 255 & 240 \\
\hline & XSs & 107 & 77 & 29 & 6 & 658 & 428 & 265 & 73 & 232 & 247 & 235 \\
\hline & Nubar & 14 & 6 & 5 & 0 & 154 & 100 & 44 & 7 & 33 & 57 & 45 \\
\hline & PFNS & 8 & 3 & 2 & 0 & 153 & 56 & 20 & 7 & 11 & 17 & 13 \\
\hline \multirow[t]{4}{*}{ E-VII.1 } & Summed & 79 & 78 & 41 & 9 & 781 & 457 & 393 & 119 & 194 & 218 & 335 \\
\hline & XSs & 74 & 77 & 40 & 9 & 668 & 415 & 388 & 118 & 188 & 203 & 329 \\
\hline & Nubar & 17 & 7 & 6 & 0 & 189 & 123 & 54 & 9 & 41 & 71 & 55 \\
\hline & PFNS & 17 & 5 & 3 & 1 & 319 & 117 & 39 & 14 & 25 & 36 & 26 \\
\hline \multirow[t]{4}{*}{$\mathrm{J}-4.0 \mathrm{u} 1$} & Summed & 78 & 64 & 24 & 7 & 636 & 394 & 238 & 85 & 179 & 211 & 272 \\
\hline & XSs & 77 & 64 & 24 & 7 & 621 & 387 & 236 & 84 & 177 & 208 & 270 \\
\hline & Nubar & 9 & 4 & 3 & 0 & 97 & 64 & 28 & 4 & 21 & 36 & 28 \\
\hline & PFNS & 5 & 2 & 1 & 0 & 96 & 36 & 17 & 5 & 6 & 10 & 10 \\
\hline \multirow[t]{4}{*}{ S-6.2rev8 } & Summed & 69 & 72 & 40 & 7 & 748 & 442 & 386 & 112 & 182 & 204 & 327 \\
\hline & XSs & 64 & 71 & 39 & 7 & 653 & 405 & 380 & 111 & 176 & 188 & 321 \\
\hline & Nubar & 17 & 7 & 6 & 0 & 188 & 122 & 54 & 9 & 40 & 71 & 55 \\
\hline & PFNS & 14 & 5 & 3 & 0 & 260 & 94 & 37 & 13 & 19 & 29 & 24 \\
\hline
\end{tabular}

\section{Nuclear data covariances}

In a recent publication [9] referred as "Comments on covariance data of JENDL-4.0 and ENDF/B-VII.1", the latest versions of the JENDL/4.0u and ENDF/B-VII.1 covariance data have been analysed. This report concluded that those evaluation of the covariance data had not yet matured or converged on the satisfactory level in their applications. In this section, we provide a comparison of nuclear data uncertainties (relative standard deviation (RSD) in \%) for CIELO files (CIELO-1=ENDF/B-VIII and CIELO-2=JEFF-3.3) and the latest JENDL-4.0 and ENDF/B-VII.1 evaluations. In addition, SCALE-6.2rev8 covariance has been also included in this analysis.

- ENDF/B-VIII.085(CIELO-1). The Beta-5 version of ENDF/B-VIII.0 files includes complete covariance matrices of the cross-sections, nubar, mubar and PFNS (at different energies, thermal, fast and high). The CIELO project is coordinated by the Nuclear Energy Agency/Working Party on Evaluation Cooperation (NEA/WPEC) Subgroup 40 since 2013. CIELO-1 cross-section data have been adopted by the ENDF project (https://www-nds.iaea.org/CIELO/).

- JEFF-3.3T4 (CIELO-2). It is the latest test JEF-3.3 neutron library produced via an international collabora- tion of Data Bank participating countries under the auspices of the NEA Data Bank. The efforts of JEFF in ${ }^{235} \mathrm{U},{ }^{238} \mathrm{U}$ and ${ }^{239} \mathrm{Pu}$ are also part of the collaboration in CIELO project. These files are the CIELO-2 set of crosssection data [1].

- SCALE-6.2. The latest SCALE-6.2.rev8 library released in 2017 which contains covariance data for 402 materials is based on ENDF/B-VII.1 and SCALE-6.1. This library includes some important changes of ENDF/B-VII.1 data such as nubar ${ }^{239} \mathrm{Pu}$ and ${ }^{235} \mathrm{U}$. The covariance library is given in 56 and 252 group energy structure in COVERX binary format. COGNAC utility code is used to convert COVERX files between binary and ASCII (https:// www.ornl.gov/scale).

- ENDF/B-VII.1 released in 2011 is largely aimed at incorporating covariance associated with a large number of nuclei and reactions, 190 materials (184 basically complete). The library is performed either using lowfidelity techniques or more robust methods relying on both experimental and model calculations; the three major actinides are evaluated with high fidelity (http:// www.nndc.bnl.gov/endf/b7.1/).

- JENDL-4.0 released in 2005 provides the data for 79 actinides. After 2005, JENDL-4.0 updated files (JENDL$4.0 \mathrm{u})$ are available for nuclides whose nuclear data are 
Table 3. Impact of different ${ }^{239} \mathrm{Pu}$ nuclear data covariances in ICSBEP suite averaged for fissile material and spectrum.

\begin{tabular}{llllll}
\hline & Fiss. mat. & \multicolumn{3}{c}{ PU } \\
\cline { 3 - 5 } & Spect. & Fast & Inter. & Mixed & Therm. \\
& Benchm. & 152 & 4 & 9 & 601 \\
& Exp. unc. & 368 & 505 & 606 & 371 \\
\hline ENDF/B-VIII.0b5 & Summed & 893 & 1550 & 1108 & 1157 \\
& XSs & 856 & 1538 & 1047 & 1059 \\
& Nubar & 214 & 146 & 232 & 333 \\
PFNS & 120 & 85 & 275 & 259 \\
JEFF-3.3T4 & Summed & 572 & 1555 & 1050 & 967 \\
& XSs & 240 & 1451 & 749 & 520 \\
& Nubar & 412 & 462 & 446 & 463 \\
ENDF/B-VII.1 & PFNS & 287 & 182 & 580 & 558 \\
& Summed & 438 & 577 & 475 & 608 \\
& XSs & 409 & 561 & 368 & 493 \\
& Nubar & 76 & 93 & 117 & 166 \\
JENDL-4.0u1 & PFNS & 120 & 85 & 276 & 260 \\
& Summed & 527 & 513 & 563 & 689 \\
& XSs & 448 & 473 & 348 & 493 \\
& Nubar & 189 & 123 & 82 & 78 \\
PFNS & 182 & 131 & 434 & 404 \\
& Summed & 343 & 572 & 465 & 605 \\
& XSs & 305 & 556 & 357 & 489 \\
& Nubar & 76 & 92 & 118 & 176 \\
& PFNS & 114 & 85 & 273 & 256 \\
\hline
\end{tabular}

partly revised from important and/or trivial error(s) (http://wwwndc.jaea.go.jp/jendl/j40/update/).

Each of the following figures shows the RSD in $\%$ of a certain reaction cross-section. JEFF-3.3T4 and ENDF/BVIII.0 $\beta 5$ have been processed with the code NJOY2012.99 in BOXER format in 238 energy groups. ENDF/B-VII.1 and JENDL-4.0u covariances are taken directly from NEA database in BOXER section which nubar and PFNS data are processed with NJOY2012.99 for this work. The SCALE-6.2 covariance file in 56 energy groups has been imported into a new JANIS database.

Figures $1-3$ show the most important nuclide reactions that contribute to $k_{\text {eff }}$ uncertainty. Five different evaluations are shown in each figure.

\section{Checking and processing}

The latest beta release JEFF-3.3 neutron library, JEFF3.3T4, contains 562 files, of which 447 files including covariances. The complete set of covariances is described as follows, MF31: 50 files, MF32/MF33: 446 files, MF34: 359 files, MF35: 35 files and MF40: 286 files. For the three major actinides, ${ }^{235} \mathrm{U},{ }^{238} \mathrm{U}$ and ${ }^{239} \mathrm{Pu}$, their covariances are evaluated using microscopic data and nuclear models. There are three nuclear data exceptions with reduced uncertainties based on the adjustment to criticality benchmarks in the fast energy region, ${ }^{235} \mathrm{U}$ (nubar) and ${ }^{239} \mathrm{Pu}$ (n,fission) and (nubar). For the ${ }^{233} \mathrm{U}$, neither PFNS covariances nor total nubar (only prompt and delayed) are given in the library.

Firstly, the pre-checking of ENDF6 format has shown some inconsistencies between MF2 and MF32 in 40 files. The processing is performed with NJOY2012.99. Only 2 files with problems were found: ${ }^{39} \mathrm{Ar}$ which one of the l-state in MF32 mismatch the value given in MF2; and ${ }^{9} \mathrm{Be}$ a cause of the problem in NJOY2012.99 to process MT values in the $875-890$ range. Both problems were solved during this work [14].

NJOY2012.99 is used to generate four different BOXER files for MF31, MF32/33, MF34 and MF35. These files can be merged into a unique BOXER file to be added into the JANIS database. The 238 energy-group is selected as the energy structure to generate covariances, because this energy structure is the most common energy structure of the $k_{\text {eff }}$ sensitivity profiles in DICE. As an example of the use of NJOY2012.99 to process covariance of PFNS, Figure 4 shows the input to produce covariance 
Table 4. Impact of different ${ }^{233} \mathrm{U}$ nuclear data covariances in ICSBEP suite averaged for fissile material and spectrum.

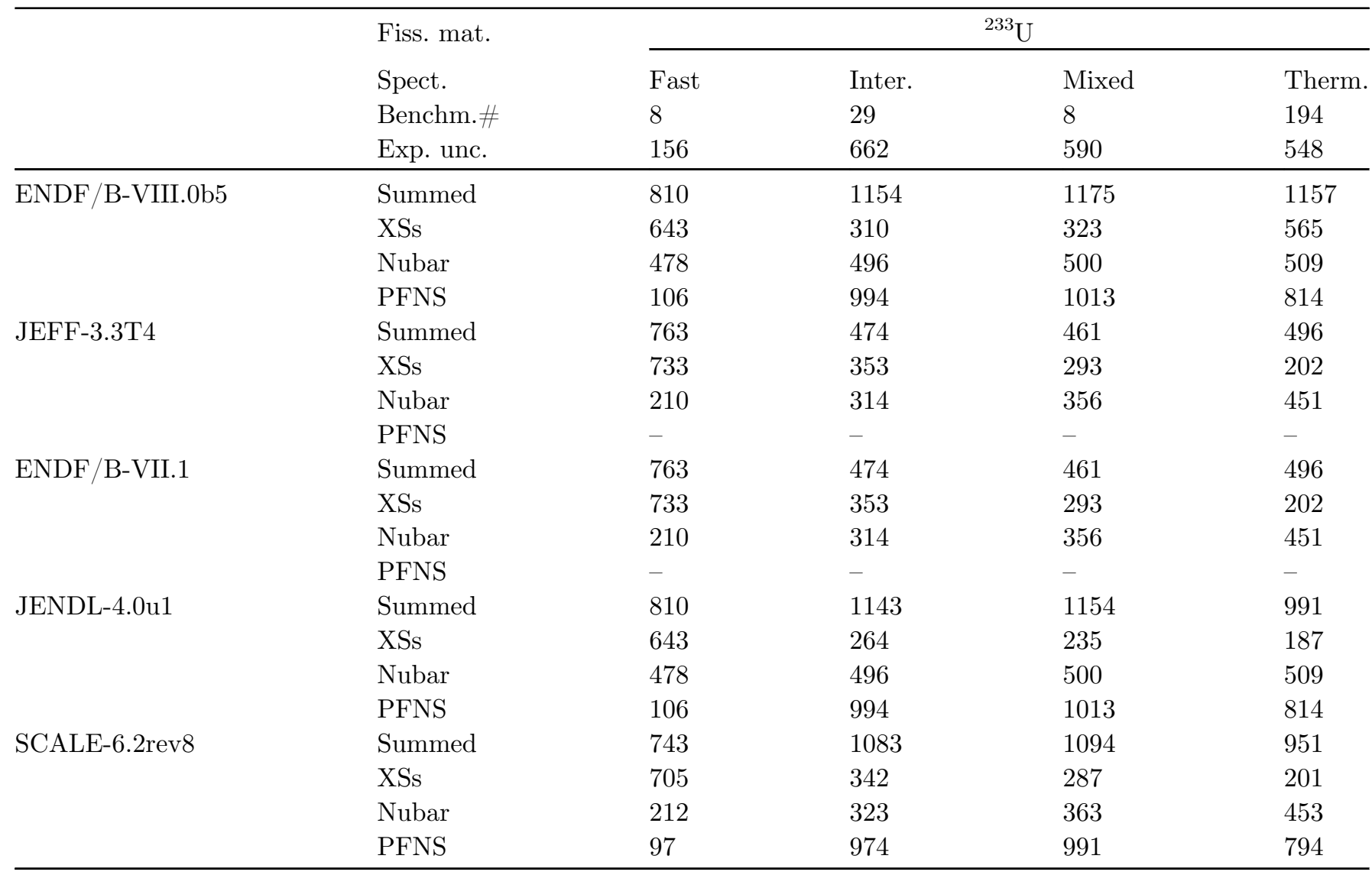

boxer files for ${ }^{239} \mathrm{Pu} / \mathrm{JEFF}-3.3 \mathrm{~T} 4$. Figure 5 is an example of different RSD in $\%$ of PFNS for ${ }^{239} \mathrm{Pu} / \mathrm{JEFF}-3.3 \mathrm{~T} 4$ as a function of different mean incident neutron fission energy.

A special NJOY input is needed to process cross-section covariance in JEFF-3.3T4 files with only MF32 section (e.g. ${ }^{35} \mathrm{Cl},{ }^{37} \mathrm{Cl},{ }^{231} \mathrm{~Pa},{ }^{233} \mathrm{~Pa}$ and ${ }^{241} \mathrm{Am}$ ). See Figure 6 for ${ }^{231} \mathrm{~Pa}$ 's NJOY input.

LAMDA code [15] is applied to the full BOXER files generated to identify non-positive definite matrices, only 30 matrices with negative eigenvalues were found, of a total number of 6931. Large uncertainties values are also checked to identify potential problems either in the evaluation or in the processing step. None of these problems were found in matrices relevant for criticality uncertainty assessment.

\section{Verification of nuclear data covariances}

The goal of this section is to assess the completeness of covariance files and the performance of these nuclear data uncertainties in a safety and criticality assessment. NDaST tool is bringing together the existing capabilities of both DICE and JANIS, to quickly propagate the impact of nuclear data covariances to criticality benchmarks. Generally speaking, the comparison of propagated nuclear data uncertainties against evaluated experimental uncertainties will give a good idea of the performance of the nuclear data. In addition, the comparison of propagated nuclear data uncertainties among nuclear data evaluations will permit to assess the completeness, disagreements and potential deficiencies of nuclear data covariances.

In order to more easily facilitate the analysis, NDaST shows graphically in a plot the $k_{\text {eff }} \mathrm{C} / \mathrm{E}$ values (in red), experimental uncertainties (in blue) and the propagated nuclear data uncertainties (in green). Figure 7 shows these values for the selection of $\mathrm{FAST} / \mathrm{Pu}$ benchmarks in the Mosteller suite [2] using the JEFF-3.3T4 library. In this Figure 7 , the nuclear data uncertainties only take into account the ${ }^{239} \mathrm{Pu}$ nuclear data uncertainty.

NDaST predicts the propagated uncertainty for a given nuclide and reaction combination. For the FAST $/ \mathrm{Pu}$ benchmarks of Figure 7, in JEFF-3.3T4 the most important contributors to the $k_{\text {eff }}$ uncertainty are fission $(\sim 300 \mathrm{pcm})$, nubar $(\sim 400 \mathrm{pcm})$ and PFNS $(\sim 360 \mathrm{pcm})$. The contribution for elastic and inelastic cross-section uncertainties are smaller, $\sim 60$ and $\sim 100 \mathrm{pcm}$, respectively. 


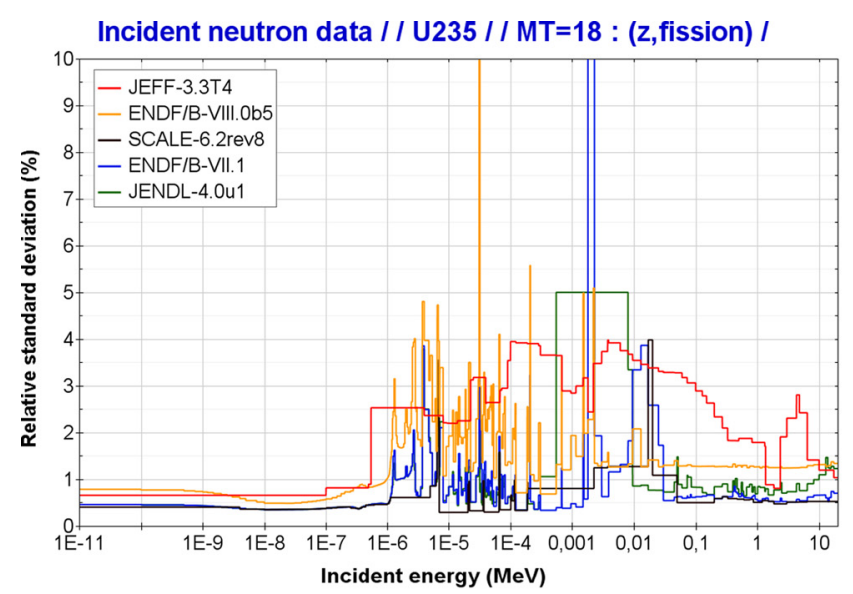

(a) ${ }^{235} \mathrm{U}(\mathrm{n}, \mathrm{f})$

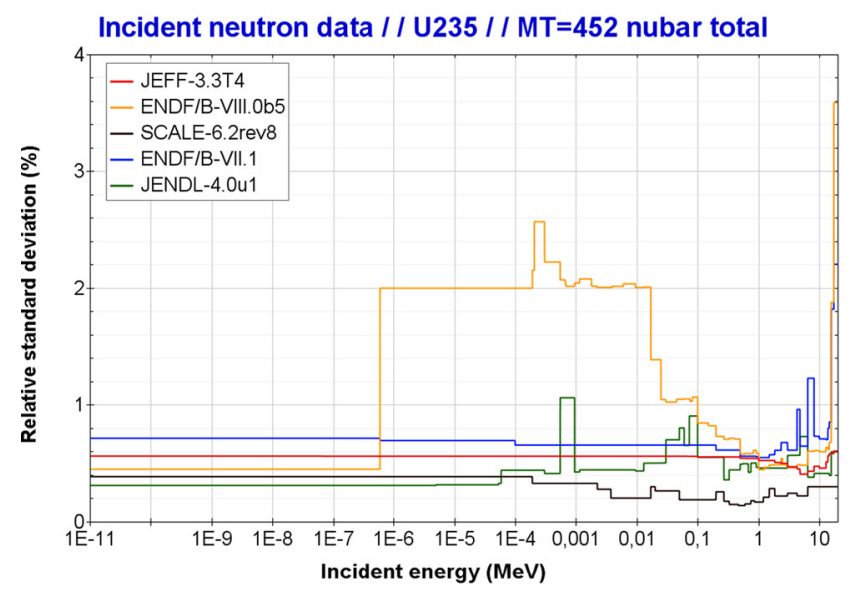

(c) ${ }^{235} \mathrm{U}($ nubar $)$

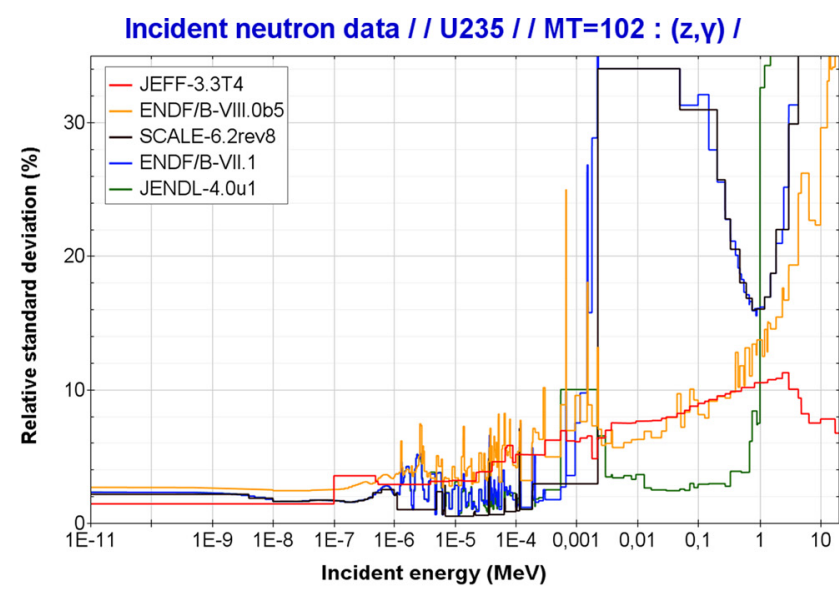

(b) ${ }^{235} \mathrm{U}(\mathrm{n}, \gamma)$

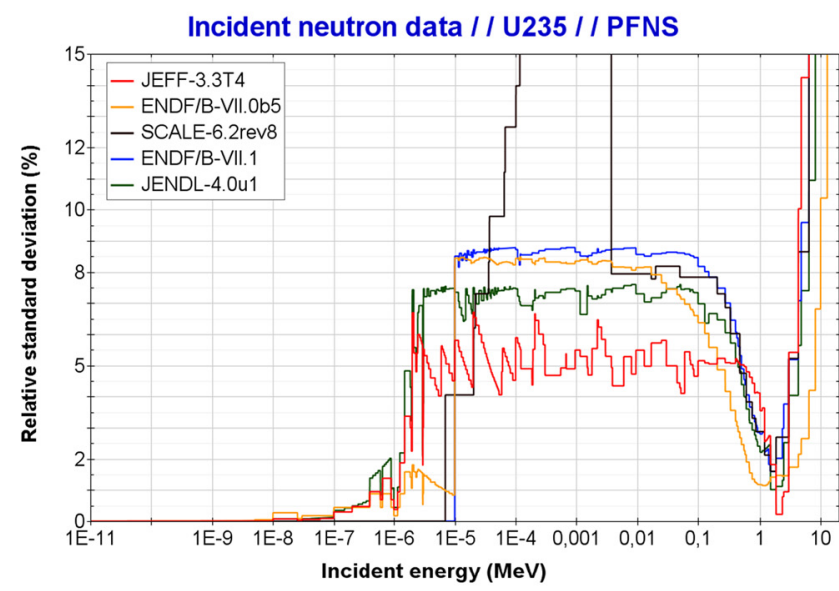

(d) ${ }^{235} \mathrm{U}(\mathrm{PFNS}), \overline{E_{\text {in }}}$ fission energy $=1.0 \mathrm{MeV}$

Fig. 1. Relative standard deviation (\%) for ${ }^{235} \mathrm{U}$.

However, other nuclear data evaluations returned highest averaged values of uncertainty due to elastic and inelastic cross-sections, ENDF/B-VIII.0 $\beta 5$ ( 300 and $\sim 540 \mathrm{pcm})$, ENDF/B-VII.1 ( 290 and $\sim 540 \mathrm{pcm})$ and JENDL-4.0u1 ( $\sim 125$ and $\sim 170 \mathrm{pcm})$.

Besides ${ }^{239} \mathrm{Pu}$ in $\mathrm{FAST} / \mathrm{Pu}$ cases, other nuclides contribute to increase the $k_{\text {eff }}$ uncertainty. As an example, the JEFF-3.3T4 top contributors in PMF9-1 benchmark are ${ }^{27} \mathrm{Al}(703 \mathrm{pcm}),{ }^{239} \mathrm{Pu}(532 \mathrm{pcm})$ and ${ }^{240} \mathrm{Pu}(182 \mathrm{pcm})$. For PMF8-1 benchmark are ${ }^{239} \mathrm{Pu}(609 \mathrm{pcm}),{ }^{232} \mathrm{Th}$ $(296 \mathrm{pcm})$ and ${ }^{240} \mathrm{Pu}(180 \mathrm{pcm})$. For the Mosteller suite, we have identified the most important contributor by isotope/element and by benchmark case for the JEFF3.3T4 nuclear data evaluation. The following is a list of these results by element: ${ }^{16} \mathrm{O}$ in HSI1-1: $367 \mathrm{pcm},{ }^{27} \mathrm{Al}$ in PMF9-1: 703 pcm, Fe in PMF26-1: 439 pcm, Cu in HMI6-4: 506 pcm, W in PMF5-1: 605 pcm, Zr in UCT1-3: 234 pcm, ${ }^{233} \mathrm{U}$ in UMF1-1: $884 \mathrm{pcm},{ }^{23} 5 \mathrm{U}$ in HMI6-4-1: $1564 \mathrm{pcm}$,
${ }^{238} \mathrm{U}$ in IMF7-1: $970 \mathrm{pcm},{ }^{239} \mathrm{Pu}$ in PCI1-1: $2097 \mathrm{pcm},{ }^{240} \mathrm{Pu}$ in PMF2-1: $834 \mathrm{pcm},{ }^{241} \mathrm{Pu}$ in PST18-9: $472 \mathrm{pcm}, 1 \mathrm{H}$ in UST8-1: $1302 \mathrm{pcm}$ and ${ }^{2} \mathrm{H}$ in HSI1-1: $2958 \mathrm{pcm}$.

Tables 1-4 give the averaged uncertainty (in pcm) in $k_{\text {eff }}$ calculated with NDaST in the ICSBEP benchmark suite for each type of fissile material and neutron spectrum. Nuclear data covariances for the four major isotopes $\left({ }^{233} \mathrm{U}\right.$, ${ }^{235} \mathrm{U},{ }^{238} \mathrm{U}$ and $\left.{ }^{239} \mathrm{Pu}\right)$ are propagated with DICE sensitivities and then averaged for the number of bechmarks of each type of fissile material. PFNS covariances are assumed at $1 \mathrm{MeV}$ incident neutron fission energy.

The averaged experimental $k_{\text {eff }}$ value is shown after the number of Benchmarks for comparison.

The following is a brief summary of the findings and conclusions based on the results shown in Tables 1-4:

- SCALE-6.2 shows lower uncertainties in $\mathrm{Pu}$ cases because the low value of nubar around $0.2 \%$ (similar to ENDF/B-VII.1). High uncertainties are found in HEU 


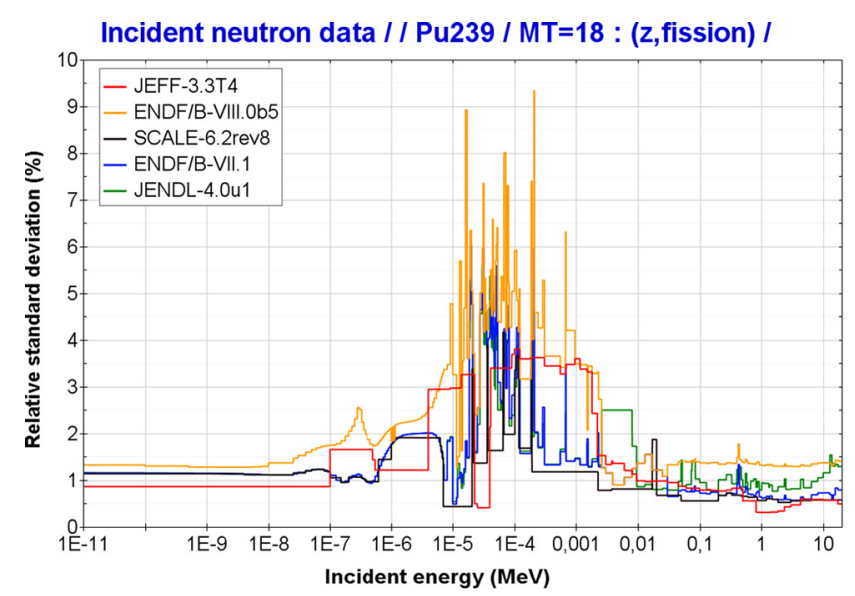

(a) ${ }^{239} \mathrm{Pu}(\mathrm{n}, \mathrm{f})$

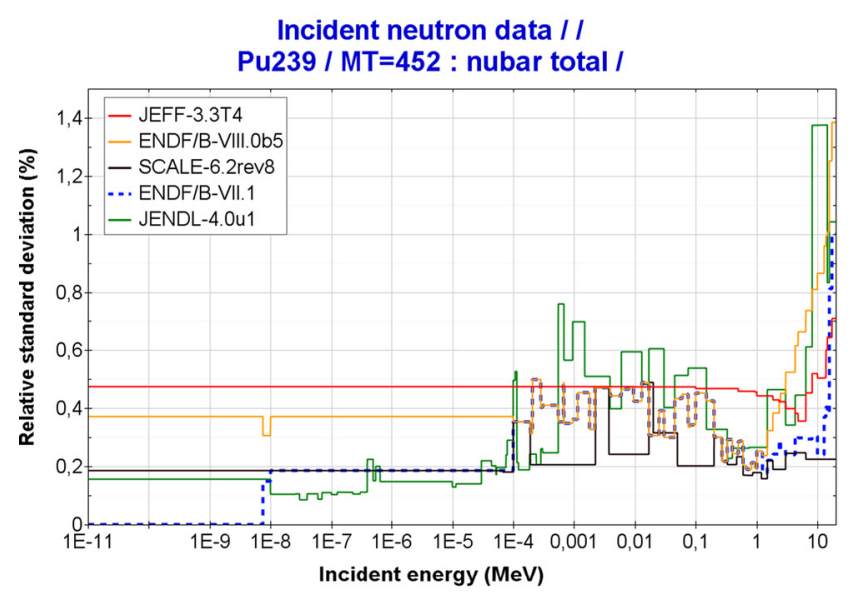

(c) ${ }^{239} \mathrm{Pu}$ (nubar)

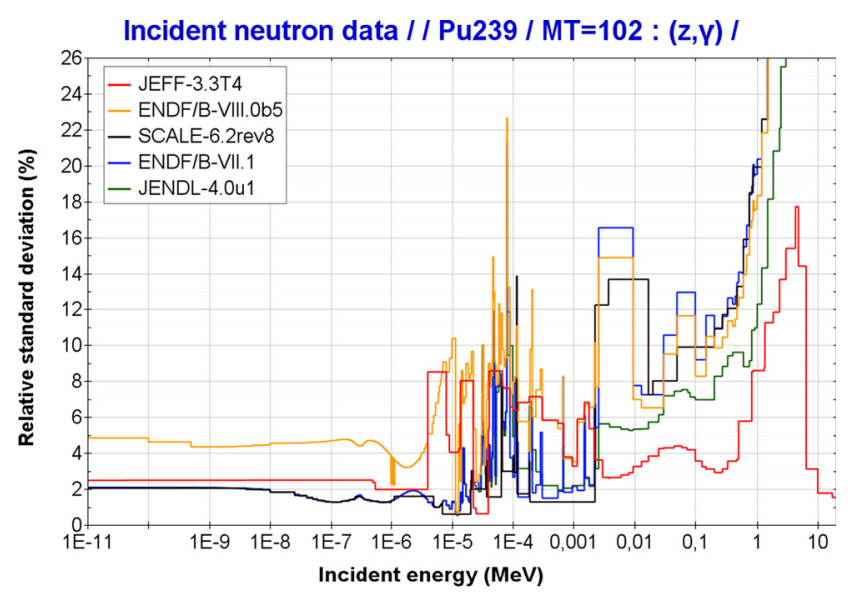

(b) ${ }^{239} \mathrm{Pu}(\mathrm{n}, \gamma)$

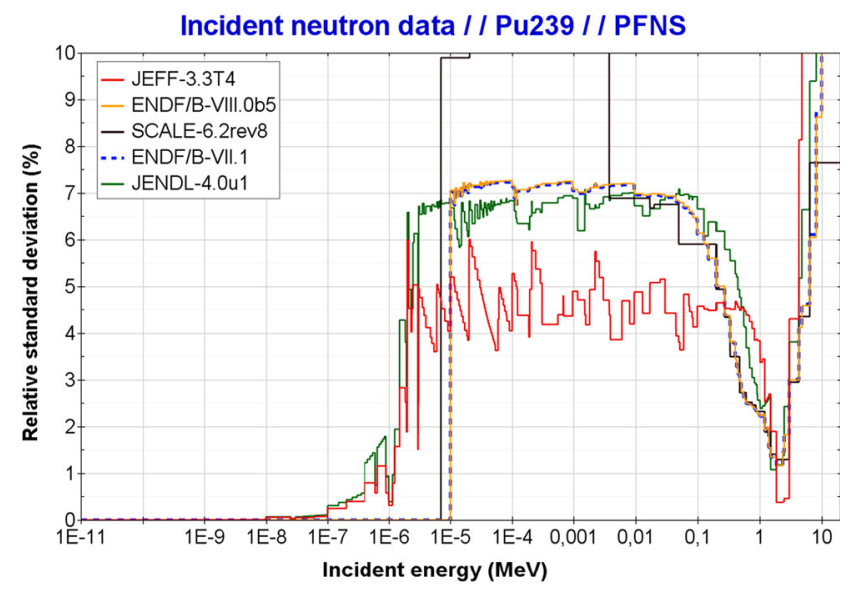

(d) ${ }^{239} \mathrm{Pu}(\mathrm{PFNS}), \overline{E_{\text {in }}}$ fission energy $=1.0 \mathrm{MeV}$

Fig. 2. Relative standard deviation (\%) for ${ }^{239} \mathrm{Pu}$.

and IEU cases because the high ${ }^{235} \mathrm{U}(\mathrm{n}, \gamma)$ uncertainty in kev-MeV energy range. Also to be remarked the high contribution to $k_{\text {eff }}$ uncertainty of ${ }^{235} \mathrm{U}(\mathrm{PFNS})$ and ${ }^{233} \mathrm{U}$ (PFNS).

- In JEFF-3.3T4, the lack of uncertainty in ${ }^{233} \mathrm{U} / \mathrm{PFNS}$ gives the lower values for ${ }^{233} \mathrm{U}$ cases (=ENDF/B-VII.1). The high uncertainty for ${ }^{235} \mathrm{U}$ (n,fission) in $\mathrm{keV}-\mathrm{MeV}$ provokes the largest uncertainties in HEU-IEU for FAST-INTERM neutron spectrum, 1100-1400 pcm. For PU case, the intermediate spectrum shows the highest uncertainty $\sim 1500 \mathrm{pcm}$, as a consequence of the high uncertainty in ${ }^{239} \mathrm{Pu}$ (n,fission) and nubar.

- JENDL-4.0u shows the higher uncertainties in ${ }^{233} \mathrm{U}$ cases because the higher uncertainties in ${ }^{233} \mathrm{U}$ nubar and PFNS. For PU-thermal and mixed neutron spectrum cases, it can be seen the impact of large uncertainty in JENDL- $4.0 u{ }^{239} \mathrm{Pu}(\mathrm{PFNS})$.

- For ENDF/B-VIII.0 $\beta 5$, it is very significant the large uncertainty in ${ }^{235} \mathrm{U}$ nubar, around $1 \%$ in the keV energy range which produces $\sim 1100 \mathrm{pcm} k_{\text {eff }}$ uncertainty in the
HEU and IEU/intermediate neutron spectrum cases. A comparison with ENDF/B-VII.1 in Table 1 shows lower uncertainty contribution for ${ }^{235} \mathrm{U}$ PFNS and cross-sections. In addition, the uncertainty ${ }^{238} \mathrm{U}(\mathrm{n}, \gamma)$ is significantly smaller in CIELO-1 giving lower uncertainty in LEU and IEU cases. ${ }^{239} \mathrm{Pu}$ fission and nubar uncertainties are increased in ENDF/B-VIII.0 $\beta 5$, it has a large impact in $\mathrm{PU} /$ INTER benchmarks. The ${ }^{233} \mathrm{U}$ uncertainties for nubar and PFNS are taken from JENDL-4.0u.

Tables 1-4 show large values of the nuclear data uncertainty in comparison with the experimental uncertainty. In general, much more accurate criticality calculations are predicted with the evaluated librarires to match low $|\mathrm{C}-\mathrm{E}|$ values. For instance, JEFF-3.3T4, for a set of 2233 ICSBEP benchmarks, it gives around $50 \%$ of benchmarks within $1 \sigma$ experimental uncertainty and $90 \%$ of benchmarks within $1 \sigma$ nuclear data uncertainty. It shows the too-wide range of nuclear data covariances. 


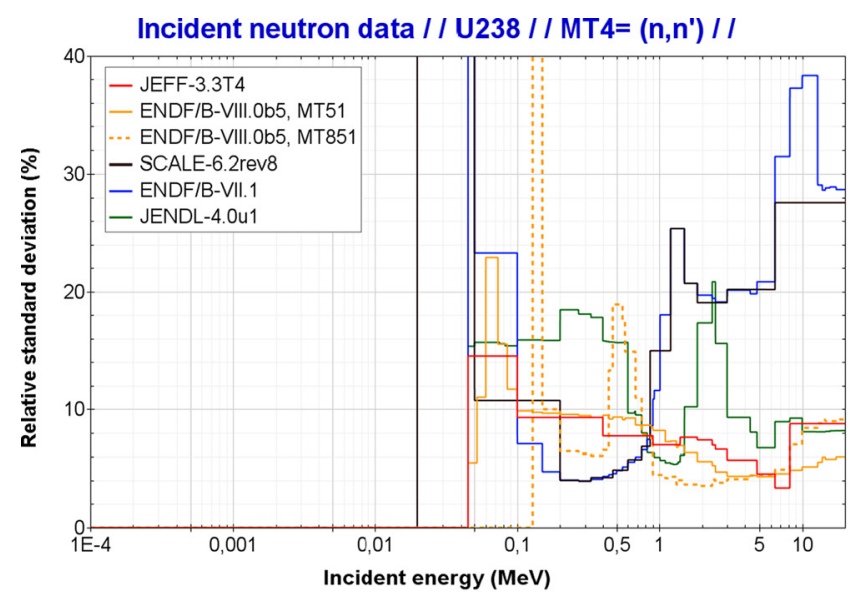

(a) ${ }^{238} \mathrm{U}(\mathrm{n}$,inel. $)$

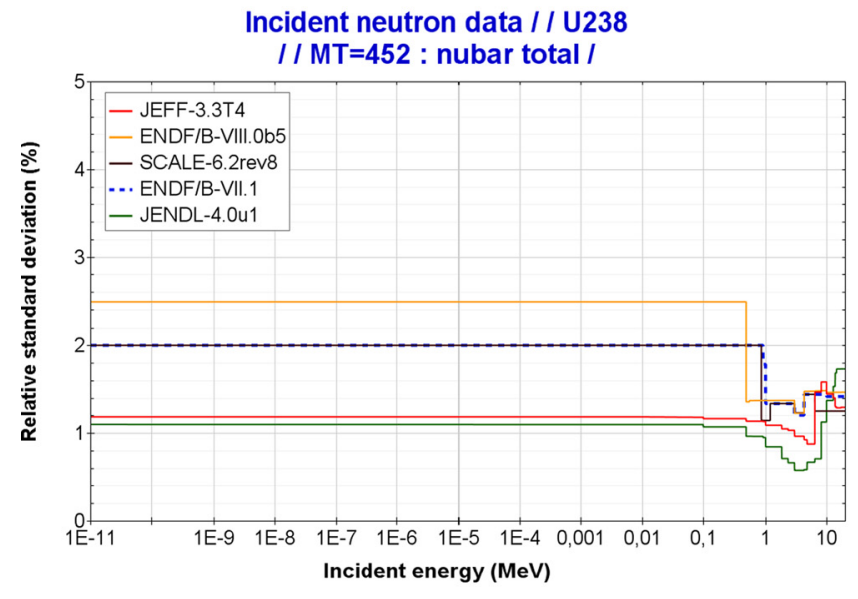

(c) ${ }^{238} \mathrm{U}$ (nubar)

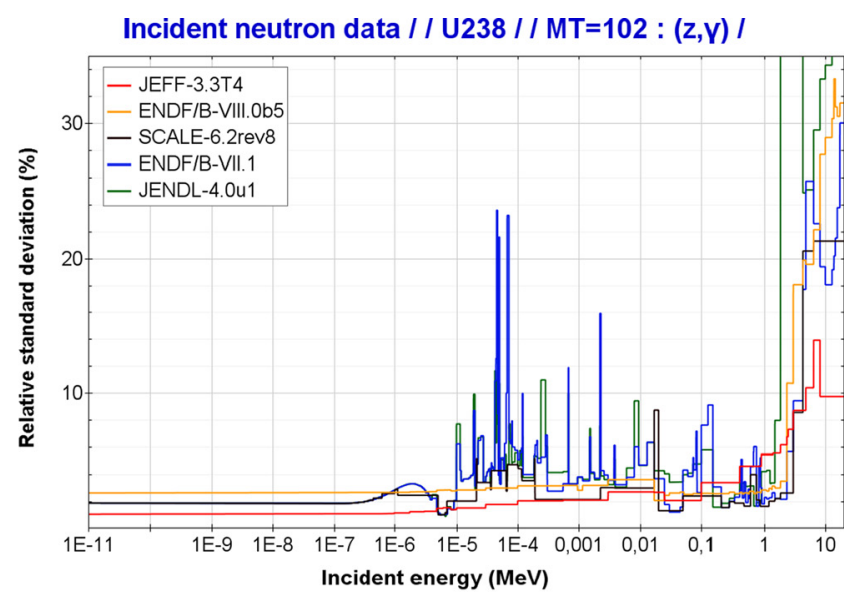

(b) ${ }^{238} \mathrm{U}(\mathrm{n}, \gamma)$

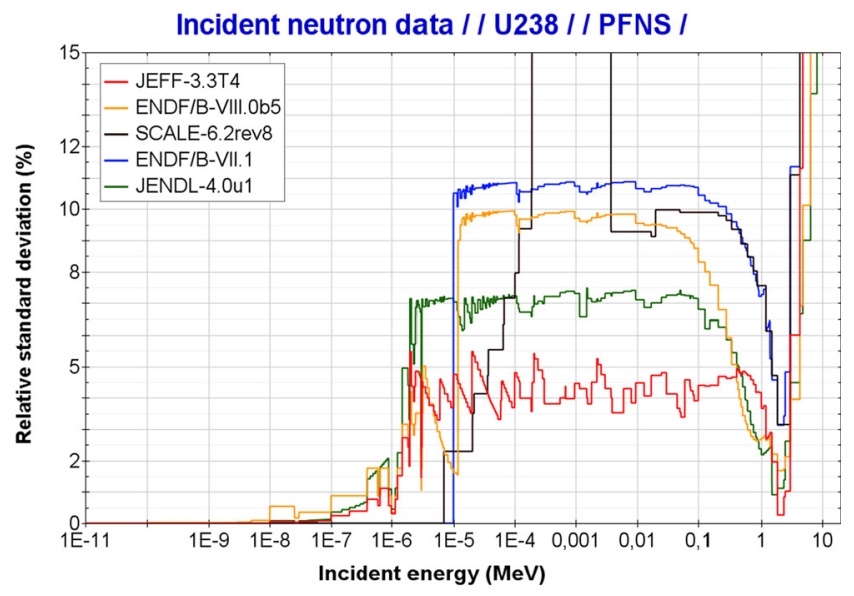

(d) ${ }^{238} \mathrm{U}(\mathrm{PFNS}), \overline{E_{\text {in }}}$ fission energy $=1.0 \mathrm{MeV}$

Fig. 3. Relative standard deviation (\%) for ${ }^{238} \mathrm{U}$.

\section{Conclusion}

JEFF-3.3T4 contains 447 files with covariance of average number of neutrons per fission (MF31), resonance parameters (MF32), cross-sections (MF33), angular distributions of elastic scattering (MF34), prompt fission spectrum (MF35) and radionuclide production (MF40). These covariances have been checked (e.g. ENDF6 format) and processed with NJOY2012.99. The verification of these covariances have been performed with mathematical (e.g. identifying negative eigenvalues) and physical (e.g. comparison with other evaluations) procedures, as well as quantifying the impact of these covariances in criticality uncertainty analysis.

Future developments in DICE/NDaST will permit to use covariance data for angular distributions. Other important feature is to extend the analysis to shielding benchmarks which will permit to quantify the impact of these covariances in other applications.

Recently, two new projects coordinated by the NEAWPEC have been initiated: (i) Subgroup 46 on "Efficient and Effective Use of Integral Experiments for Nuclear Data Validation" [16] to define the methodology for verifying the physical properties of nuclear data covariances based on Adjustment methodologies; (ii) Subgroup 44 on "Investigation of Covariance Data in General Purpose Nuclear Data Libraries" [17] to provide guidance to the international community on methods for systematic and consistent evaluation of covariance data for the whole energy range. This underlines that defining credible nuclear data uncertainties remains still a challenging problem for the nuclear data community [18]. 


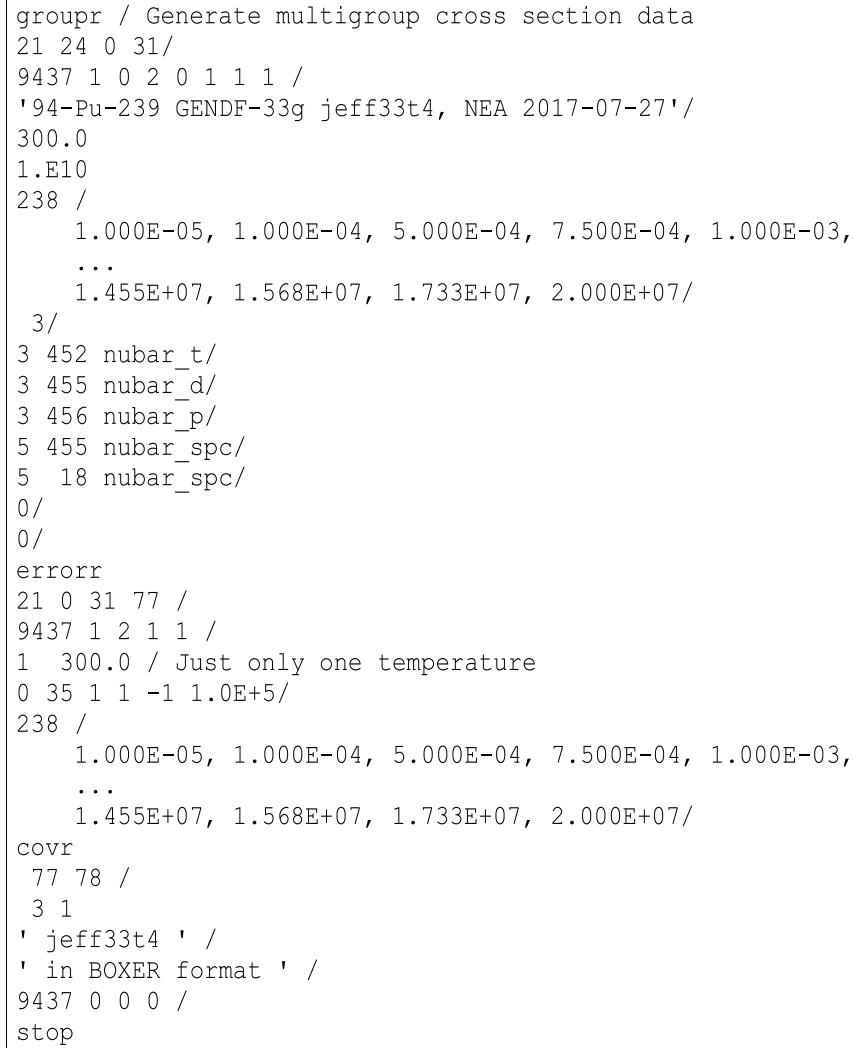

Fig. 4. An example of NJOY input to process MF35/PFNS covariance.
Incident neutron data I I MAT9437 I MT=18 : (z, fission) / Covariances data (BOXER) Relative standard deviation

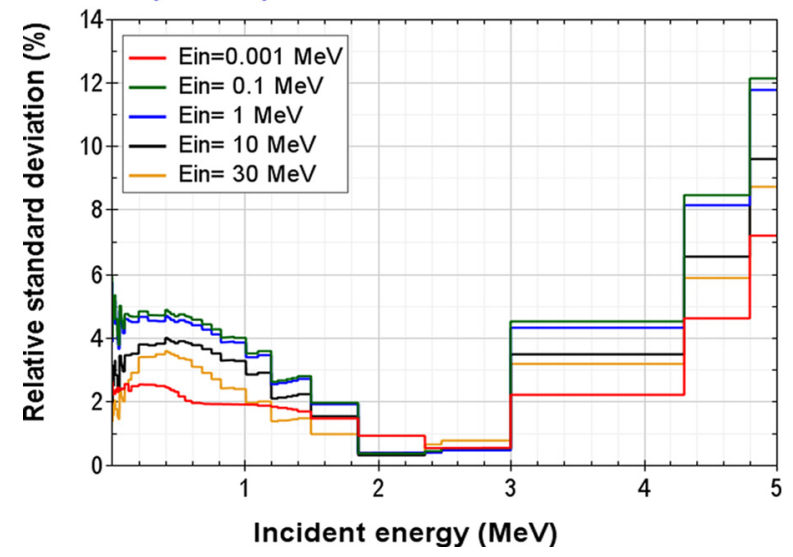

Fig. 5. RSD in \% of ${ }^{239} \mathrm{Pu} / \mathrm{JEFF}-3.3 \mathrm{~T} 4 \mathrm{PFNS}$ distributions as a function of mean incident neutron fission energy.

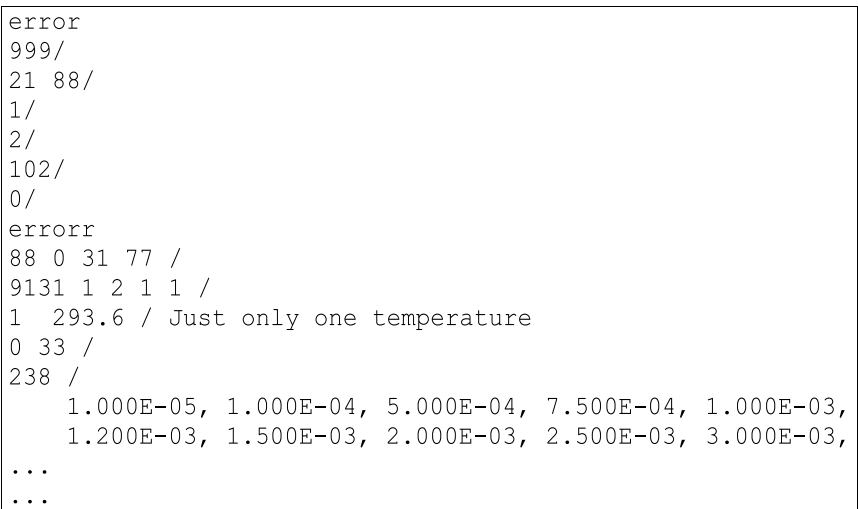

Fig. 6. NJOY input to process files with only MF32.

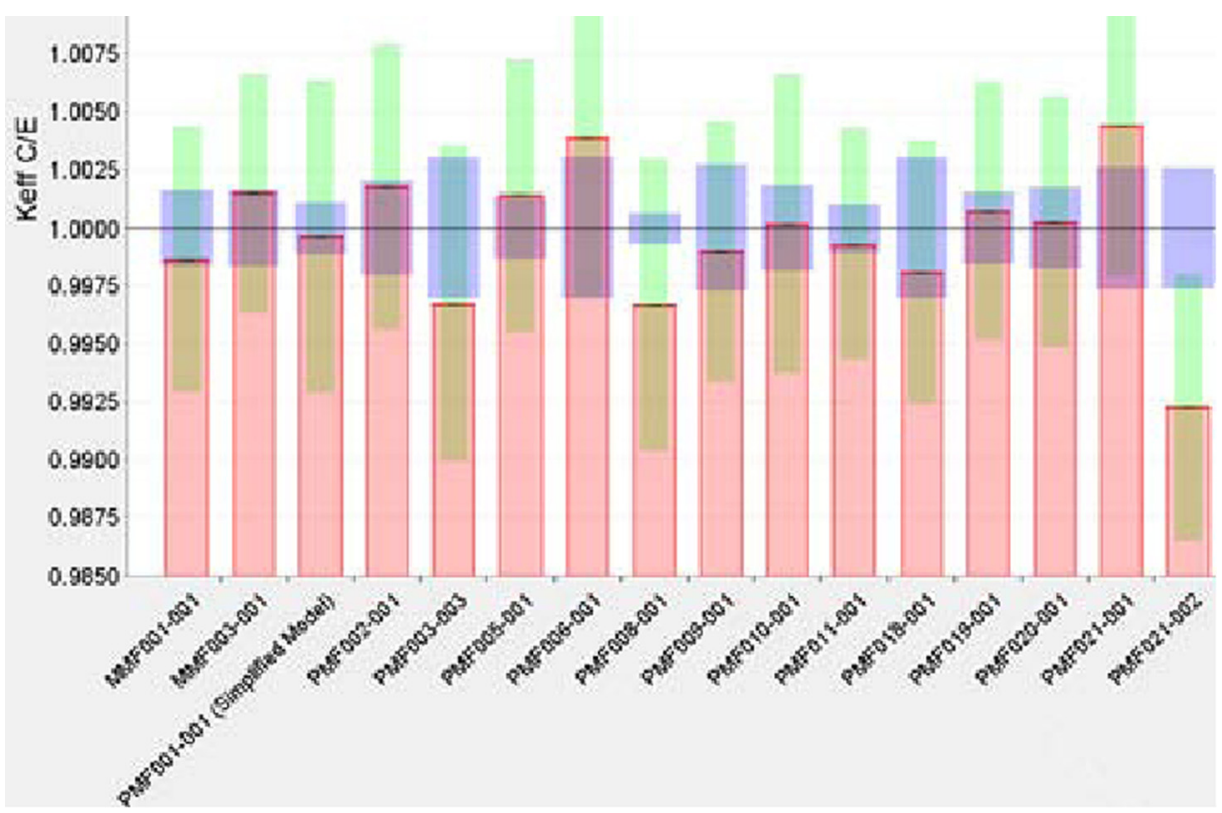

Fig. 7. NDaST output plot, $k_{\text {eff }} \mathrm{C} / \mathrm{E}$ (in red), experimental (in blue) and propagated nuclear data uncertainties (in green) due to ${ }^{239} \mathrm{Pu}$ covariance of JEFF-3.3T4. 


\section{Author contribution statement}

O. Cabellos and J. Dyrda conceived the present work. O. Cabellos carried out the first part of the work on checking and processing nuclear data covariance. O. Cabellos and J. Dyrda performed the verification of nuclear data uncertainties assessing the impact on keff uncertainty in the ICSBEP benchmark suite. O. Cabellos and J. Dyrda supervised the main findings of this work. N. Soppera updated JANIS and NDaST tools, new capabilities for visualization and computing were applied in this work. O. Cabellos wrote the manuscript with support from J. Dyrda and N. Soppera.

\section{References}

1. JEFF-3.3T4, http://www.oecd-nea.org/dbdata/jeff-beta/ JEFF33T4/
2. O. Cabellos et al., EPJ Web Conf. 146, 06004 (2017)

3. J. Dyrda, O. Cabellos, JEFF/DOC-1840 (2017)

4. O. Cabellos, JEFF/DOC-1773 (2016)

5. M.B. Chadwick et al., Nucl. Data Sheets 112, 2887 (2011)

6. K. Shibata et al., J. Nucl. Sci. Technol. 48, 1 (2011)

7. B.T. Rearden, WPEC/SG44 meeting (2017)

8. M.B. Chadwick et al., EPJ Web Conf. 146, 02001 (2017)

9. WPEC/SG39 Intermediate Report, NEA/NSC/R (2016)

10. A.C. Kahler, NJOY2012.82, LA-UR-12-27079 (2017)

11. J. Dyrda et al., EPJ Web Conf. 146, 06026 (2017)

12. N. Soppera et al., EPJ Web Conf. 146, 07006 (2017)

13. C.J. Diez et al., EPJ Web Conf. 146, 02026 (2017)

14. NJOY2012.99, https://www.oecd-nea.org/dbprog/njoy-links. html

15. I. Kodeli, NEA-1798 ANGELO-LAMBDA

16. G. Palmiotti, WPEC/SG46 meeting (2017)

17. V. Sobes, WPEC/SG44 meeting (2017)

18. M. Chadwick, CIELO Collaboration, Nucl. Data Sheets, to be published (2018)

Cite this article as: Oscar Cabellos, James Dyrda, Nicolas Soppera, Checking, processing and verification of nuclear data covariances, EPJ Nuclear Sci. Technol. 4, 39 (2018) 